\title{
Learning from Forced Completion vs the Option to Opt Out: An Experiment on a Hybrid of the Game of 21 and the Centipede Game
}

\author{
Timothy Flannery \\ Missouri State University \\ TFlannery@missouristate.edu
}

\author{
Cara Sibert \\ Missouri State University \\ Sibert911@live.missouristate.edu
}

January 8, 2020

\begin{abstract}
This study examines how the option to opt out facilitates learning in the Game of 21. It compares the performance of subjects in the Game of 21 and a Hybrid Centipede21 Game where players can opt out of the game at any time and receive a decreasing payment for doing so as the game progresses. Additionally, the experiment introduces a novel concept of a "dumb computer" that always makes suboptimal decisions. Performance against the "dumb computer" determines the level of foresight of subjects in order to compare the amount of learning between the Hybrid Centipede-21 Game and the traditional Game of 21 . Results indicate players drop out strategically and earlier as the game progresses with a few ending immediately as the backward induction solution predicts; however, contrary to our predictions, players learn better when forced to finish the game, particularly women. Some of the difference in learning occurs due to a small set of players giving up when they have the option to opt out while the rest may be attributed to the larger role strategic uncertainly plays or the additional complexity of the game.
\end{abstract}

Keywords: Backward Induction, Learning, Optimal Strategy, Centipede Game, Game of 21, Race Game

JEL Classifications: C73, C91, D83 


\section{Introduction}

Previous studies demonstrate that many people display limited foresight by only looking ahead a few periods instead of solving the problem from the end as backward induction requires (Dufwenberg, Sundaram, and Butler 2010; Gneezy, Rustichini, and Vostroknutov 2010). Given the many important long-term decisions in life, such as retirement, career choices, and mortgage obligations, society must search for methods to better teach the process of backward induction and improve long-term decision-making. Our experiment tests whether the ability to opt out improves learning in a multi-period game. Opting out effectively breaks large decisions up into smaller ones, allowing someone with limited foresight to at least make the optimal decision near the end of the game.

To test the hypotheses that opting out facilitates learning, we design a hybrid version of the Centipede Game and Game of 21, referred to as C21 hereafter, and compare the amount of learning from it to the traditional Game of 21, referred to as G21. C21 allows subjects to drop out at any time and still receive earnings, with the amount of earnings decreasing as the game progresses. Because G21 requires many levels of foresight and contains a dominant strategy, it is perfect for testing how much subjects learned. In C21, strategically opting out not only increases earnings but should also make discovering the optimal strategy simpler since pairs of players only need enough foresight to plan from the drop out point in the previous round. Thus, agents should learn better and more quickly in C21 compared to G21. Additionally, our design contributes to the literature on cognitive reasoning tests by using a "dumb computer" that provides a better estimate of how many steps players learned to think ahead 1

The experimental results provide insight on public policy with applications to education, retirement, and healthcare. Opting out is relevant for health benefits, known for its annual enrollments, by permitting employees to exit health plans for new ones partway through the year. Furthermore, better foresight should also lead to improved financial decision-making, particularly regarding retirement. Foresight is important in education, such as how students select their major and when students can drop out of courses. The dropout dates provide time for students to reevaluate their progress in a course and either create a plan of action to improve their grade or drop out if one realizes the desired grade is unachievable.

Our experiment contains the novel cognitive test to measure a foresight using "indicated rationality" (Dufwenberg et al. 2010). Each subject plays a computer that sub-optimally responds and allows the player to use the dominant strategy path of play on the following turn. Thus, even if participants fail to play perfectly, they can exhibit some level of backward induction. Though computers use optimal strategies in several studies $2^{2}$ and Cardella 2012 makes computers randomly choose, we know of no other study with computers programmed

\footnotetext{
${ }^{1}$ All measures in these games are noisy since a player sometimes select the optimal choice by luck.

${ }^{2}$ The following studies are some of the studies which use an optimizing computer in their design: Cardella in G21, Johnson, Camerer, and Rymon 2002 in a bargaining game, Flannery and Roberts 2018 in a principalagent setting, Hoppe and Schmitz 2015 in an adverse selection environment, and Bayer and Renou 2016 in a game requiring iterated reasoning.
} 
to sub-optimally decide. Subjects play this "dumb computer" at the beginning and end of the experiment to measure the improvement in foresight since subjects enter the lab with varying levels of foresight.

Even though subjects drop out strategically and sooner as the rounds progress, players, especially women, appear to learn better when forced to finish. Slower learning in C21 may occur due to its greater complexity from an additional option or due to the larger importance of strategic uncertainty since whether to opt out or not depends on opponent quality. Additionally, some discouraged players drop out in the winning position, referred to as giving up, which likely slows learning. However, in C21, almost as many players learn the optimal strategy, and some subjects even play the subgame perfect equilibrium of dropping out immediately by the last few rounds. Our results suggest that quitting itself does not harm subjects, but the option to quit causes a subset of players to give up and makes the game slightly more difficult by providing an additional option that requires one to understand the quality of one's opponent when used optimally. As for the upside of quitting, the lowest paid player per pair earned more on average when the option to quit existed. Thus, quitting seems to hinder learning if players quit when they fail to understand something, essentially giving up, but it has the positive effect of mitigating losses for a failed cause (knowing one will lose in our experiment).

\section{The Centipede Game and Game of 21}

Our experiment utilizes two traditional games testing backward induction: G21 and the Centipede Game. The Centipede Game, introduced by Rosenthal (1982), challenges the power of backward induction as a solution concept. Dufwenberg et al. (2010) and Gneezy, Rustichini, and Vostroknutov (2010) introduced "race games" and G21, respectively, to test backward induction, since they have dominant strategies.

In the Centipede Game, players sequentially choose whether to continue or opt out of the game. The subgame perfect equilibrium of most versions has players end the game each turn, and, hence, it should end immediately. However, in many Centipede Game experiments, players rarely stop immediately (McKelvey and Palfrey 1992; Fey, McKelvey, and Palfrey 1996), reasoning that continuing may be rational given the uncertainty about an opponent's rationality. Continuing could also send a (possibly false) signal regarding one's own rationality to persuade the opponent to continue next turn. Our study mitigates these effects by pairing players for the entire experiment to reduce uncertainty about opponents' abilities. While one concern with the Centipede Game is that players may rely on altruistic tendencies and continue the game to increase total surplus (Fey et al. 1996), we eliminate the effect of efficiency preferences by using a constant sum payoff to C21.

Unlike the Centipede Game, G21 contains a dominant strategy, so strategic uncertainty plays no role, and the game's constant sum nature prevents efficiency concerns from influencing decision-making. G21 is a two-player, sequential game employing several iterations of 
the format $\mathrm{G}(\mathrm{k}, \mathrm{m})$ with $\mathrm{k}$ representing the maximum amount a subject can increase their position and $\mathrm{m}$ the winning number $3^{3}$ For this experiment, the value of $\mathrm{k}$ is 2 (a player increases the previous number by 1 or 2 ). The value of $\mathrm{m}$ is 21 (a player wins when choosing a value of $\mathrm{k}$ that sums to 21). The first mover begins by choosing either 1 or 2 , and, after observing that choice, the second mover selects a number either 1 or 2 higher than the number of the first mover. For example, if the first mover chooses 1, the second mover chooses 2 or 3 . If the first mover chooses 2 , the second mover chooses 3 or 4 . Players continue this pattern of adding 1 or 2 to the previous number until a player reaches 21 and wins. Solving G21 requires a simple application of backward induction. To reach 21, players choose 18, so that their opponent chooses 19 or 20 . Reaching 18 requires one to reach 15, and so forth in multiples of 3. Players likely recognize the importance of hitting larger multiples of 3 such as 18 before earlier ones like 3, which require further reasoning. Because 3 is the smallest multiple of 3, G21 contains a second-mover advantage since the first-mover cannot win, unless the second-mover blunders. Therefore, as second-movers against a computer, subjects should win by choosing each multiple of 3, referred to as key numbers, if they understand the solution.

Several tests confirm G21 is long enough that most players initially lack foresight to recognize the optimal strategy and simple as it requires no high-level computation. Demographic characteristics may suggest individuals with certain characteristics are predisposed to learn the game quicker and better, such as through gender (Padden Hall, 2009; Brosig-Koch, Heinrich, and Helbach, 2015). Brosig-Koch et al.'s 2015 research suggests teams perform better at inductive reasoning through G21 and win more than individuals. Furthermore, cognitive ability such as college entrance exams and IQ tests or the ability to apply K-level thinking to assess and predict the level of strategic sophistication of other players in the P-Beauty Contest game may be related to how a player performs in G21 (Carpenter, Graham, and Wolf, 2013; Dufwenberg, et al., 2010; Gneezy, et al., 2010). However, research also suggests that while these demographic factors influence the ability to backward induce, characteristics such as age prove that the ability to backward induce can be taught (Brosig-Koch, et al., 2015). In Cardella's 2012 experiment on G21, learning-by-doing is more effective than learning-by observing.

G21 provides valuable insight on how people often have limited foresight, and improving foresight has the potential to significantly improve the well-being of individuals given the importance of dynamic decision-making in everyday life. Importantly, Dufwenberg et al. 2010 suggest participants can transfer knowledge from one game to another, so backward induction can be learned and used in other applications, such as education. For example, one of the authors of this paper recently attended a meeting discussing a new initiative "finish in four" to help students graduate in four years.4 Many students fail to recognize the need to take an average of 15 credits as 12 credits is considered full time while others miss required classes only offered once a year. By applying backward induction, students

\footnotetext{
${ }^{3}$ Gneezy et al. (2010) use this notation and refer to such games as "race games."

${ }^{4}$ It should be noted the initiative does not push students to graduate in four years, but rather helps students who want to recognize the proper way to do so.
} 
can create their four year schedule starting from the end to ensure they graduate in four years.

\section{Experimental Design}

The subject pool consisted of undergraduates from Missouri State University. A total of 132 subject: ${ }^{5}$ participated in 13 sessions during March and April of 2019. We used SOPHIE software (Hendricks, 2012) for programming and ORSEE software for recruiting and scheduling (Greiner, 2015). Funding came from Missouri State University's Student Initiative Fund. Each subject received a $\$ 5$ show-up payment in addition to experimental earnings. We used a between subject design where subjects either played C21 or G21. In each session, subjects read instructions on the computer with an interposed quiz before making any decisions and received paper instructions at the start of the experiment 6

To start, participants in both treatments played G21 as second movers against a computer. The computer sub-optimally responds by avoiding multiples of 3 and randomly responds when the human player selected a multiple of 3 previously. Therefore, if subjects skip a multiple of 3, they can select one on the next turn, except if moving from 19 to 20. This design allows the subject to exhibit signs of backward induction without having to play perfectly. For example, if a player fails to choose 3, the earliest key number, they can choose 6 and the remaining key numbers to reach 21. Players know they play against a computer but never learn its decision process. Unless players move from 19 to 20 instead of choosing 21, they should defeat the "dumb computer". Subjects received 63 ECUs (where 30 ECUs equals \$1) for defeating the "dumb computer", the same amount earned for beating humans. The results against the "dumb computer" in the first round indicate how well a player backward induced and whether any players knew the optimal strategy before entering the laboratory. Though similar to naïve opponent of Cardella 2012, our "dumb computer" contains one key difference: Cardella's artificial opponent is programmed to randomly add one or two while ours always plays sub-optimally.

Our paper contributes to the literature by providing a cognitive measure on foresight by pioneering a technique of a computer that always plays sub-optimally instead of one that plays optimally or randomly. Several recent papers 7 use the game of "Hit 15", a race game with $k=3$ and $m=15$, in studies to measure the foresight of a subject. As far as we know, these papers have subjects play optimizing computers multiple times. Our "dumb computer" has three primary advantages: it only requires a single game for an adequate measure of foresight, it can be used multiple times since most players will not learn the optimal strategy by playing once, and players cannot learn by mimicking as they can against

\footnotetext{
${ }^{5} 133$ subjects actually participated. One subject entered the lab twice using a second university email, and we omitted his second round data as well as the data of the person with whom he paired.

${ }^{6}$ See appendix for both sets of written instructions.

${ }^{7}$ Burks, Carpenter, Goette, and Rustichini (2009) introduced "Hit 15" as a cognitive measure. Other papers to use it include Altmann, Falk, and Wibral (2012); Carpenter et al (2013); and Cueva and Rustichini (2015).
} 
an optimizing computer. We believe learning the optimal solution through discovery instead of mimicking indicates a subject has a richer understanding of the solution.

After playing the "dumb computer", subjects were randomly matched with the same, anonymous, opponent and for ten games 8 Our program randomly generated the turn order each match. Each match divided up 63 ECUs between the pair of subjects. After the ten matches, subjects again played G21 against the "dumb computer". We used 12 matches (10 with a person and 2 with the "dumb computer") to ensure participants played enough for learning to occur.

Playing the "dumb computer" at the end of the experiment allows us to measure each player's improvement. Dufwenberg et al 2010 measure a player's indicated rationality-the smallest multiple of three chosen when each succeeding number is a multiple of three. Our analysis uses the average positive change in indicated rationality per pair similar to indicated rationality defined by Dufwenberg et al 2010. We look at positive changes as some players perform worse against the "dumb computer" because they did well in the first round by chance. Performing worse due to participating in the experiment seems unlikely, so if a player, for example, played a perfect game by chance against the "dumb computer" but later skips the number 12 , we calculate this as a change of zero instead of a negative. Because the play of one player in a pair is not independent of another, we use the average change per pair instead of per player. We also compare the amount of games against the "dumb computer" with perfect play at the end to see if the ability to opt out facilitates learning as expected. Below is a description of G21 and C21.

\subsection{Control: G21}

The control group plays G21 described earlier with no opt-out option. The player reaching 21 wins and receives a payoff of 63 ECUs while the loser receives nothing. A screen-shot of the second turn is in the appendix.

\subsection{Treatment: C21}

The treatment introduces a game similar to the one described above but incorporates a property of the Centipede Game. Due to this property, players split a constant-sum of 63 ECUs, divided according to the game number where a player chooses to end the game. At any point, a player can choose to end the game and receive a payout according to the table in the appendix. To minimize the effect of equity preferences, C21 begins with an unequal

\footnotetext{
${ }^{8}$ We employed fixed matching for two reasons. First, we wanted to minimize uncertainty about partners' ability; if players are uncertain about the other player's ability, they may react differently, and spend the round attempting to ascertain information about the other player. Secondly, we wanted to minimize the effects of high-ability players. If a player knows the strategy entering the round, they may contaminate multiple results if they are partnered with different subjects each time by showing each player the optimal strategy.
} 
payoff, 42 and 21 ECUs. Player one moves first and can choose to continue the game by increasing the number 0 by 1 or 2 , or to end the game by opting out and taking a payoff of 21 ECUs. If Player 1 opts out immediately, 42 ECUs goes to Player 2, and the game ends. If Player 1 continues the game, and moves the game number up one (two) then one (two) ECU(s) moves (move) from one payoff to the other [21, 42 changes to 20, 43; (19, 44)], and then Player 2 chooses. Player 2 can now opt out or select the smaller payoff, the larger payoff goes to the non-opt out player, or continue the game by increasing the game number by one or two more ${ }^{9}$ If Player 2 continues and move the game number up one (two), an (two) ECU(s) moves (move) to the bigger payoff and Player 1 chooses again. This process continues until a player ends the game or reaches the number 21, with the player reaching the number 21 receiving all 63 ECUs. ${ }^{10}$

The game has a unique backward induction solution where the first player opts out immediately, earning the first mover 21 and the second 42. However, opting out is sub-optimal if a player believes one's opponent will select a non-key number. Furthermore, players may skip key numbers early on and fail to opt out if they do not recognize an analytical solution exists and believe that winning occurs by chance. Fixed matching increases the likelihood players know the capabilities of one's opponent, and, therefore, increases the possibility players drop out early compared to a design with random re-matching.

\section{Hypotheses}

We predict that the opt-out option causes players to learn better and more quickly compared to G21 where they must finish the game. We include four hypotheses:

Hypothesis 1. Players opt out earlier and more often in C21 in later rounds than in earlier rounds.

In previous work on G21, Dufwenberg et al. 2010 suggest that players can recognize 18 as a key number, and in many cases 15. In the first few matches, we expect players to opt out if the other player selected 18 on the prior turn. However, as players gain experience, they start to understand the importance of hitting earlier key numbers $(3,6,9,12,15)$ and opt out sooner to earn more. Near the end of the experiment, second movers should know every key number and that their payoff for continuing is always higher than the opt-out option, regardless of the other player's strategy. First-movers should recognize that they will lose (if the second mover selects optimally) and opt out immediately.

\footnotetext{
${ }^{9}$ See the appendix for a screen shot of the second turn in this game.

${ }^{10}$ Other studies also modify G21 but use teaser treatments and nudges, which are composed of monetary incentives placed on key numbers within the Game of 21, have been proven to accelerate learning of the game, when provided mid-game on the equilibrium path (Padden-Hall, 2009; Parsons, 2016; Neilson, et al, 2016). The model of the Centipede Game suggests that providing monetary incentives may expedite learning by encouraging players to opt out and recognize the optimal strategy.
} 
Hypothesis 2. In C21, matches end less often on 21 and non-multiples of three than they end on key numbers.

Players will avoid letting the game end on 21 as they realize they earn more from dropping out at 18 since almost all opponents will assuredly win at that point. Moreover, they will opt out after the other player states a key number, like 15, as opposed to dropping out when the previous player selects a non-key number like 16 . When players discover the optimal strategy and go first, that player will likely opt out immediately to earn 21 ECUs.

Hypothesis 3. Players in C21 will play the dominant strategy more frequently than players who play G21 in the final round, improve their play more, and make fewer errors in their choices.

Players might recognize their mistakes and end the game once they understand the importance of key numbers in C21. However, lacking the option to opt out, players may struggle to recall their choices in previous matches, complicating the task of backward induction. With the ability to opt out, players will likely remember when they (or their opponent) opted out. Thus, the key number may remain salient in their memory, and they realize the importance of hitting the next smallest key number. Players who opt out effectively shorten the game for the next round and eliminate a step in backward induction. In G21, players must remember the importance of all key numbers, a seemingly more difficult feat.

One can visualize how opting out can improve decision-making with a game tree. If players foresee one move ahead, they should end the game at 18 in the first round. Once players comprehend the importance of 18 , they effectively "chop off" the pieces of the game tree after 18 since each player will drop out at that point. Players should then realize they must select 15 to reach 18 even if they have limited foresight for the same reasons they saw the importance of 18. Given both players drop out at 15, players can "chop off" the pieces of the game tree after it. This process continues until players drop out immediately as predicted by the SPE. By the end, the subjects should realize reaching each multiple of three guarantees victory.

In addition to comparing each player's performance against the "dumb computer" between G21 and C21, we incorporate the concept of "total error rate" by counting the proportion of key numbers that players skip throughout rounds as a measure of understanding, introduced by Gneezy, et al., 2010 and used by Cardella 2012. That is, if a subject has the opportunity to choose a key number, and skips it, such as going from 5 to 7 or 17 to 19 , the subject has indicated a level of sub-optimal reasoning (2012). To analyze the choices of key numbers, we sort choices into three categories: correct, error, or impossible. A correct move occurs from selecting a key number, such as moving from 4 to 6 while an error occurs when skipping a key number, such as moving from 4 to 5 . Finally, an impossible choice happens when a player lacks the opportunity to move to a key number, such as moving from 3 to either 4 or 5 . We define total error rate to be the frequency of errors, divided by the sum of errors and correct choices. We hypothesize that players in the sessions of $\mathrm{C} 21$ will have a lower total error rate. In C21, we do not consider failing to drop out on a key number as an error since 
such a strategy may be optimal if one believes an opponent does not yet fully understand the solution to the game; however, we classify dropping out at non-key numbers like 2 as an error.

Hypothesis 4. Players in C21 will learn the dominant strategy faster than those in G21.

To determine which group learned faster we use the concept implied epiphany rounds (similar to a measure used by Dufwenberg et al 2010 called epiphany delay). Subjects undergo an implied epiphany round when they never choose a dominated strategy in the current round and any succeeding rounds. In G21 and C21, this amounts to selecting every multiple of 3 when feasible.

\section{Results}

\subsection{Hypothesis 1}

Figure 1 shows the data supports Hypothesis 1 since the average point players dropped out decreases over time. If players failed to drop out, the drop out point is listed as 21 . In the first round, players usually either failed to opt out or opted out at 18, with the average game ending at 18.73; however, by the end of the experiment, some players opted out immediately and only four pairs went all the way to 21 (though two players sub-optimally opted out at 1 and 2). In the final round, the average game ended just below 12. A two-sided Mann-Kendall test supports our hypotheses by showing the average drop out rates trend downward with a p-value of 0.0022 . Furthermore, by comparing the average drop out point for each player in the first half to the second half, a two-sided Wilcoxon Ranked Sum test also supports our hypothesis with a p-value of less than 0.00001 .

Some players seemed to recognize the backward induction solution by the end. Thus, they likely understood the optimal strategy themselves and believed their opponents recognized it. Only one group opted out immediately in either rounds 1 or $2^{11}$ while in the final two rounds 6 pairs out of 33 opted out immediately in each round. Of course, some of these players may have opted out because they gave up as opposed to learning the analytical solution. Such behavior likely slows learning instead of facilitating it. However, the data indicates that this seems unlikely for most who opted out immediately, except for one player who opted out immediately in round 9 but also opted out at 2 in rounds 8 and 10.12

Opting out earlier and earlier only demonstrates subjects' learning if they opt out at the correct numbers. Opting out at 18 early and 17 later demonstrates a failure to learn.

\footnotetext{
${ }^{11}$ This opting out likely occurred due to giving up rather than understanding the game as the pair had two later games where a player opted out on 1 and 17, two suboptimal points to opt out.

${ }^{12}$ Another player who opted out immediately near the end also opted out at 2 , but this player made this choice early in the game in round 3 and never opted out outside of a key number again.
} 


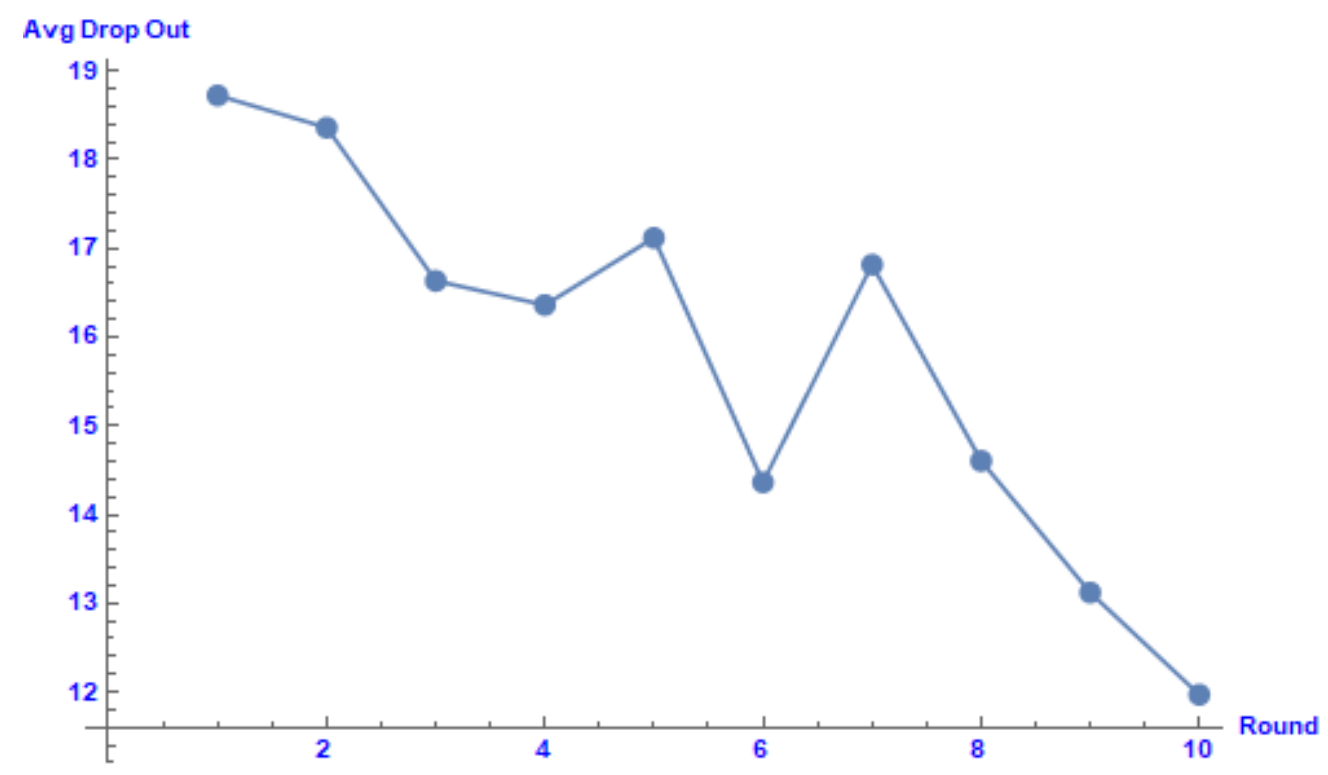

Figure 1: Average Drop Out Point in C21

\subsection{Hypothesis 2}

Table 1 lists the frequency of players dropping out on key numbers. The majority of rounds ended before 21 on a key number instead of a non-key number. The behavior is extremely consistent with Hypothesis 2 in all rounds but the first where 15 out of 33 games ended at 21. With the exception of the first round, a two-sided binomial test shows that opting out on a key number before 21 occurred with a majority of pairs ${ }^{13}$ The number of players opting outing out on key numbers grew large as the game progressed. In the second half of the experiment, no more than four pairs took the game to 21 and at most four pairs dropped out on non-multiples of three. The average amount of strategic drop outs (those on a key number) increased from an average of 24.4/33 in the first five rounds to 28.6/33 in the final five rounds. This provides evidence that players recognized the key numbers as the game progressed. Although the data strongly supports Hypotheses 1 and 2, this only provides evidence players learned how to play better in C21. We want to know whether C21 causes players to learn better than G21.

\section{Table 1: Frequency of Key Number Drop Outs}

\begin{tabular}{|c|c|c|c|c|c|}
\hline Rounds 1-5 & 0.55 & 0.70 & 0.76 & 0.88 & 0.82 \\
\hline Rounds 6-10 & 0.94 & 0.88 & 0.88 & 0.85 & 0.79 \\
\hline
\end{tabular}

\footnotetext{
${ }^{13}$ The maximum p-value of 0.0351 occurs in round 2 where 23 pairs had a player drop out on a key number and ten pairs finished the game. P-values for rounds 3-10 were about one percent or below.
} 


\subsection{Hypothesis 3}

Though players learn in C21, we find no evidence to support Hypothesis 3, which states that participants in $\mathrm{C} 21$ learn better than those in G21. A perfect performance by a player (selecting all key numbers) in the final round against the "dumb computer" strongly indicates the player learned the dominant strategy ${ }^{14}$ In the final round, an participant can achieve perfect play because of the second-mover advantage. Since pairs of players do not play independently, we compare both the pairs with at least one perfect play in C21 to G21.

Overall, 18 of 33 pairs had at least one player achieve perfect play in the final round after G21 while 15 of 33 pairs did after $\mathrm{C} 21 \sqrt{15}$ Both treatments had 7 pairs where both players achieved perfect play by the end. Using perfect plays alone, no evidence exists to support that players learned better with the option to opt out.

Though perfect play demonstrates a full understanding of the game, it omits a large amount of learning by players who learned to recognize key numbers such as 12 and 15 that they failed to recognize earlier. Therefore, we compare the average positive change in indicated rationality per pair that consists of a measure of 0-7 on improvement per pair with half measures since it averages over two players. Each number of the measure indicates an improvement of play by one key number. Thus, hitting 18 as the last key number against the first "dumb computer" and 15 against the second yields a positive change in indicated rationality of 1 for that player. A measure of 7 indicates a player went from losing to the "dumb computer" in the first round to a perfect game in the final round. If one player in the pair improves by 1 and the other by 6 , the average positive change in indicated rationality for this pair is 3.5. A measure of 0 means both players in the pair performed the same or worse against the final "dumb computer" compared to the first.

Figure 2 provides the $\mathrm{CDF}$ of the positive change in indicated rationality per pair between those in G21 and C21. The average positive change in indicated rationality per pair was 2.65 after playing G21 and 2.045 after C21. A two-sided Mann-Whitney U test comparing the difference gives a p-value of 0.1085 , just out of the significant range ${ }^{16}$ Finally, in $\mathrm{C} 21$ 7/33 had a positive change in indicated rationality per pair of zero, meaning neither player improved, significantly different from G21 with only 1/33 using a two-sided Fisher's Exact Test with a p-value of 0.0539 . This provides some evidence that forcing completion instead of allowing players to opt out better facilitates learning, contrary to Hypothesis 3, especially at the bottom of the distribution. Comparing error rates each round shows no significant difference between treatments.

To understand why players failed to learn better in C21, we examined the pairs where players gave up. We distinguish between giving up and quitting by separating the decisions

\footnotetext{
${ }^{14}$ One player actually had perfect play against the "dumb computer" the first time but not against it the second time; therefore, perfect play does not perfectly demonstrate a player discovered the optimal strategy but such occurrences are rare.

${ }^{15} \mathrm{~A}$ two-sided Fisher's Exact Test shows this is not significant with a $p=0.6228$.

${ }^{16}$ The Kolmogorov-Smirnov gives a similar result with a D-statistic of 0.24242
} 


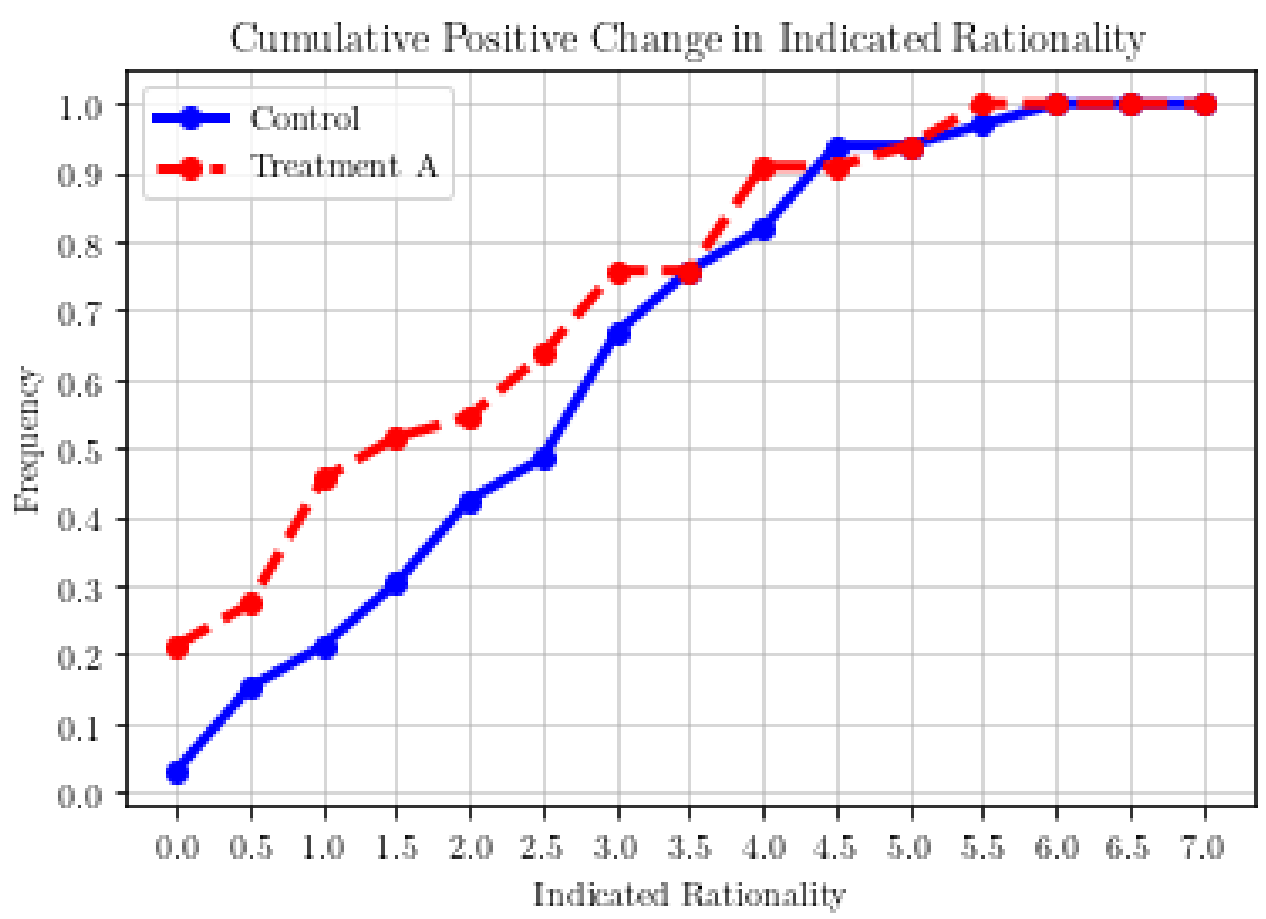

Figure 2: Cumulative Distribution of the Average Positive Change in Indicated Rationality per Pair: the blue line represents the players in the control (G21) while the red line represents the players in the treatment $(\mathrm{C} 21)$.

based on whether a participant ends on a key number, quitting, or opting out non-multiples of 3, giving up. For example, players who drop out at 2 do so because they do not understand the solution, since that player can win by selecting the remaining key numbers. Omitting the pairs where a player clearly gave up increases the average positive change in indicated rationality per pair from 2.045 to 2.278 in C21. Thus, the pairs with a player who clearly gave up attribute to some of the difference in learning when comparing C21 and G21. All six players who gave up had a lower positive change in indicated rationality: four had 0 , one had 1 , and the other had 2 for an overall average of 0.5 . The players who gave up did not seem to be playing better opponents at the beginning, and only one opponent of a player who gave up knew the optimal strategy by the end. Therefore, the reason these players chose to give up is unclear.

Looking at the indicated rationality of the final round against the "dumb computer", both the largest numerical and relative difference in the number of players for different levels of reasoning occurred at 2: with 12/66 players in C21 and 5/66 players in G21. While the reasoning of 2 (which coincides with players recognizing 18 as the last key number) has a difference of 7 players, no other level of reasoning has a difference larger than 3 people. Why did so many more people in $\mathrm{C} 21$ remain stuck on 18 without further backward inducing compared to the players in G21? From their discussions with their subjects, Gneezy et al. 2010 mention that players fail to immediately backward induct due to uncertainty of whether 
reaching the initial key number, 18, is indeed optimal and focus on the ways to reach 21 from 18 instead of realizing the importance of the next key number. C21 always has more uncertainty as the player must also consider whether to opt out at every node. Thus, the lack of better learning in C21 could occur due to the additional complexity associated with knowing whether dropping out is optimal, which depends on opponent quality.

\subsection{Hypothesis 4}

Hypothesis 4 only applies to the players who realized the optimal strategy. In G21, 25 players used the optimal strategy: 14 of them learned it with their opponent while 11 learned it while their opponent did not. The implied epiphany round, the last round where no error occurred, happened on average for the 14 pairs at 6.79 rounds and 8.64 rounds for the 11 solo learners. In C21, 22 participants played perfectly: 14 in pairs and 8 on their own. On average, the pairs learned the optimal strategy in 6.93 rounds while the players who learned it individually learned it in 8.1. Given the closeness of these numbers, C21 appears not to affect the speed of player learning.

\subsection{Effect of Opting out on Payoffs}

Though opting out appears to slightly reduce the amount of learning, particularly for those who give up, the ability to opt out may be better for some players as they lose less than when forced to finish. To explore this effect, we compared the payoffs of the lowest earning player per pair. The average for the lower earners playing C21 exceeded the lower earners of G21 at roughly 211 ECUs compared to 202 ECUs, though the difference was not significant ${ }^{17}$

Therefore, the option to drop out creates both a negative and positive effect. Dropping out reduces the amount of learning, especially those who give up. However, dropping out allows poor performing players to earn money in games they would otherwise lose.

\section{Exploratory Analysis}

To understand why subjects failed to learn better in $\mathrm{C} 21$, we reexamined the data and considered theoretical reasons for the unexpected finding, besides players giving-up. After looking over the data again, strategic uncertainty may have slowed learning in C21, particularly for women.

\subsection{Effect of Opponent on Learning}

Cardella 2012 shows that humans learn to backward induct better when playing optimizing opponents compared to naive opponents. Our data shows the initial quality of an opponent (measured using indicated rationality) does not significantly affect the performance

\footnotetext{
${ }^{17}$ Using a two-sided t-test
} 
of a player in the final round against the computer (again measured using indicated rationality). However, in C21, players learn together, unlike G21. The Spearman rank correlation coefficient between the average positive change in indicated rationality per pair gives a significant coefficient of $0.46068(p=0.00698)$ in C21 but only a coefficient of -0.03371 in G21, which is not significant $(p=0.85226) .18$

Though players learning better together in C21 than G21 seems surprising, this pattern of learning has a theoretical foundation as strategic uncertainly affects the optimal decision in C21 but not G21. In G21, the optimal choice is independent of the quality of one's opponent as one chooses a multiple of 3 when feasible. In C21, even though opting out immediately earns one the most with a rational opponent, players should remain in the game if they believe the opponent will err. Thus, players in C21 have an incentive to learn the quality of their opponent, which in turn teaches them how to improve their own play (if the opponent learns well).

Because each player's learning depends on the learning ability of the opponent in C21, those paired with players who gave up usually failed to learn. Three of the six partnered with a player who gave up had a positive change in indicated rationality of 0 . The other three players had measures of 1, 2 and 6, so only one player drastically improved who paired with a player who gave up.

The correlation between opponents learning occurring in C21 and not G21 is also consistent with the theory that the additional complexity of the game may have also contributed to worse learning. Earlier research showed that forced cognitive load, such as having to remember a phone number while playing the game, can distress one's ability to induce backward properly (Carpenter, et al., 2013), so the additional concern about an opponent's ability and the added decision of when to opt out may do the same. This additional effect appears to affect men and women's behavior differently.

\subsection{Gender}

At the end of our experiment, we asked subjects to disclose their gender in a survey to examine whether males and females performed differently ${ }^{19}$ In $\mathrm{C} 21$ and G21, the median multiple of three followed by all multiples of 3 (indicated rationality) was 18 for both genders, indicating two steps of reasoning ${ }^{20}$ Thus, our initial results with the "dumb computer" show that males and females exhibited roughly the same level of reasoning just as Cueva and Rustichini 2015 do using "Hit 15" in a study on all gender versus mixed gender markets. Though the genders displayed equal levels of reasoning initially in C21 and G21, women

\footnotetext{
${ }^{18}$ Performing the same test on indicated rationality from the final rounds per pair yields similar results.

${ }^{19} \mathrm{We}$ asked for no other demographic information.

${ }^{20}$ We include understanding 21 as the winning number as one step of reasoning while 0 steps of reasoning would be failing to understand 21 as the winning number unlike Dufwenberg et al. 2010 who consider 18 one step of reasoning. In their work all pairs have a player reach 21 while a player may not reach 21 with the "dumb computer" in our experiment, so the difference is understandable. The average number of reasoning steps were: 2.22 for females and 2.28 for males in G21, 2.62 for females and 2.92 for males in C21.
} 
appeared to learn better in G21.

In the final round against the "dumb computer", men exhibited a median of 5 and 5.5 steps of reasoning in G21 and C21, respectively, so they performed slightly better at the end of the $\mathrm{C} 21$; on the other hand, women displayed a median of 5 and 3 levels of reasoning in the G21 and C21, respectively. Thus, it appears men learn roughly the same in both games while women perform worse in the C21. Only two females clearly gave up, ${ }^{21}$ so the worse performance must be attributed to another factor, such as strategic uncertainty since it only theoretically matters in $\mathrm{C} 21$.

\section{Conclusion}

Opting out permits players to mitigate losses, recognize mistakes, and start over with improved decision-making, but, unfortunately it may slow learning. Our research shows that many players reduce their losses from dropping out when they have a low chance of success, since most players dropped out strategically. Subjects learn when given the option to quit, which we observed through earlier strategic drop outs as the game progressed as well as improved play. Unfortunately, by providing the option to quit, some subjects give up and end up worse off as they fail to learn, and, partly due to this effect, subjects learn less with the option to quit than when forced to complete the game. Whether the slower learning also occurs from additional complexity or from greater uncertainty in the game is important because if greater complexity causes slower learning, opting out likely always slows learning as it adds complexity by providing an additional option. However, if the uncertainty of the quality of one's opponent caused the slower learning in C21 relative to G21, given its theoretical importance, opting out may not slow learning in non-strategic scenarios. Finally, men appear to learn equally in both games, but women learn better in G21; thus, future research should explore whether strategic uncertainty affects the ability of men and women to learn differently.

Our study provides insight on policies related to foresight such as in education regarding drop out rates, which many universities aim to minimize. One of the reasons subjects backward induced less in C21 compared to G21 occurred because some of the subjects gave up in some rounds; thus, people should only quit when they know why they quit. For example, if students consider leaving a university to pursue a career that does not require a degree, quitting may improve their welfare. However, when students leave college because they feel "overwhelmed", these students should wonder why they feel this way before leaving. Universities and college counselors should keep this in mind when meeting with a student who contemplates dropping out (if they already do not). The same reasoning applies to those who leaving/switching health insurance plans and retirement accounts. In short, we must distinguish between quitting and giving up.

\footnotetext{
${ }^{21}$ Of the six individuals who gave up, three identified as male, two identified as female, and one identified as other.
} 
Our paper also introduces the novel idea of the "dumb computer" to use as a cognitive measure regarding a person's foresight. The "dumb computer" has many advantages over the current standard of playing an optimizing computer, most importantly, players cannot learn through mimicking. We look forward to its application in future research and the comparison of its effectiveness in measuring foresight compared to playing optimizing computers in "Hit 15".

Determining policies on quitting/dropping out remains difficult as policy makers must weigh the positive effect of the gains from those who exit a failed cause to the negative effect of some who give up and fail to learn what is best for themselves. Furthermore, the option to opt out may complicate decision-making (because of an additional choice), slowing the learning process more. More studies need to examine the effect of opting out in other environments as well as how to help people understand the process of thinking ahead via backward induction. For example, our study included a game against another human opponent, would learning occurring better in a simple decision environment with the option to opt out when the ability of an opponent is irrelevant? Given the improved outcomes from learning by doing compared to learning by observing (Cardella, 2012), perhaps subjects may learn more by teaching one another? We hope our work inspires such research and provides insight for policymakers on the best way to provide health insurance, keep students in school, and teach people to best plan for retirement.

Acknowledgments: We would like to thank Dr. Siyu Wang for providing advice and insight on our project and Dr. Stephen Roberts for helping with the programming. Additionally, we want to thank the Student Government Association at Missouri State University, who through the Student Initiative Fund, provided us the monetary resources to perform the study. 


\section{Appendices}

\section{A [Instructions for Control]}

Today you will be playing a two player sequential game. In this game, each player chooses to move the game up one or two numbers, starting from zero. The first mover starts the game by choosing the number one or two. The second mover then chooses one or two numbers above the first mover's chosen number. For example, if the first mover chooses 2, the second mover can choose either to move to 3 or 4 . The goal of the game is to be the player to reach 21. Whoever reaches 21 wins! The winner of the game earns 63 ECUs while the loser receives 0 ECUs. (Note $30 \mathrm{ECUs}=\$ 1$ )

After everyone reads the instructions and plays the game against a computer, you will be randomly matched with an opponent and play the game a ten times against that same opponent with the turn order randomly determined each round. Each of your identities will remain confidential. Once you complete a round, a screen will display your earnings for the round, and you will wait until all other pairs finish their round before starting the next round.

Make sure you always hit continue when you finish reading the text! Otherwise, you will delay the game for everyone.

Before and after everyone completes the ten rounds against their opponent, each player will compete against a computer one time. If you win against the computer, you receive 63 ECUs in compensation for each win.

Once you finish the game, you will be asked a few questions and finally taken to a screen with your overall earnings. Do not leave the screen with your final earnings until the investigator comes and verifies them. If at any time you have any questions, please raise your hand. Finally, please be respectful of other participants by remaining silent while everyone makes their decisions. Good luck! 


\section{B [Instructions for Hybrid Centipede-21 Treatment]}

Today you will be playing a two player sequential game. In this game, each player chooses to move the game up one or two numbers, starting from zero. The first mover starts the game by choosing the number one or two. The second mover then chooses one or two numbers above the first mover's chosen number. For example, if the first mover chooses 2, the second mover can choose either to move to 3 or 4 . The goal of the game is to be the player to reach 21. Whoever reaches 21 wins! The winner of the game earns 63 ECUs while the loser receives 0 ECUs. (Note $30 \mathrm{ECUs}=\$ 1$ ).

You or your opponent also have the opportunity to end the game early on every turn. Whoever ends the game early receives an amount of ECUs equal to twenty-one minus the current game number while the other player receives sixty-three minus the payment to the player ending the game early. For example, if you end the game when the game number is 5 , you will receive 16 ECUs (21 minus the game number=21-5=16) while your opponent receives 47 ECUs (63-your payment=63-16=47). Note that you can even end the game immediately if you are the first mover and receive 21 ECUs while your opponent receives 63-21=42 ECUs.

After everyone reads the instructions and plays the game against a computer, you will be randomly matched with an opponent and play the game a ten times against that same opponent with the turn order randomly determined each round. Even if a game ends early, it still counts as one of the ten games. Each of your identities will remain confidential. Once you complete a game, a screen will display your earnings for the round, and you will wait until all other pairs finish their round before starting the next round.

Make sure you always hit continue when you finish reading the text! Otherwise, you will delay the game for everyone.

Before and after everyone completes the ten rounds against their opponent, each player will compete against a computer. When you play the computer, you will not have the opportunity to end the game early. You will only be able to add one or two numbers to the game until you or the computer reaches 21. If you win against the computer, you receive 63 ECUs in compensation.

Once you finish the game, you will be asked a few questions and finally taken to a screen with your overall earnings. Do not leave the screen with your final earnings until the investigator comes and verifies them. If at any time you have any questions, please raise your hand. Finally, please be respectful of other participants by remaining silent while everyone makes their decisions. Good luck! 


\begin{tabular}{|c|c|c|}
\hline Opt-Out Number & Opt-Out Player Payoff & Non-Opt-Out Player Payoff \\
\hline 0 (Beginning of Game) & 21 & 42 \\
\hline 1 & 20 & 43 \\
\hline 2 & 19 & 44 \\
\hline 3 & 18 & 45 \\
\hline 4 & 17 & 46 \\
\hline 5 & 16 & 47 \\
\hline 6 & 15 & 48 \\
\hline 7 & 14 & 49 \\
\hline 8 & 13 & 50 \\
\hline 9 & 12 & 51 \\
\hline 10 & 11 & 52 \\
\hline 11 & 10 & 53 \\
\hline 12 & 9 & 54 \\
\hline 13 & 8 & 55 \\
\hline 14 & 7 & 56 \\
\hline 15 & 6 & 57 \\
\hline 16 & 5 & 58 \\
\hline 17 & 4 & 59 \\
\hline 18 & 3 & 60 \\
\hline 19 & 2 & 61 \\
\hline 20 & 1 & 62 \\
\hline 21 & 0 & 63 \\
\hline
\end{tabular}




\section{Game of 21 Decision Screen}

\section{SOPHIE}

The other player just added 2 to the game, so the game is currently at the following number: 4 .

Select option "1" to add one number to the game, taking the game to the number 5 or select option "2" to add two numbers to the game, taking the game to the number 6 .

Would you like to add one or two numbers to the game?

D 1: Add one number to the game

2: Add two numbers to the game

Submit 


\section{Centipede-21 Game Decision Screen}

\section{SOPHIE}

The other player just added 1 to the game, so the game is currently at the following number: 3

Select option "1" to add one number to the game, taking the game to the number 4 or select option "2" to add two numbers to the game, taking the game to the number 5 . If you want to end the game now and earn $18 \mathrm{ECUs}$, select option "E"

Would you like to add one number to the game, two numbers to the game, or end the game?

Q] 1: Add one number to the game

2: Add two numbers to the game

E: End the game

Submit 


\section{References}

[1] Altmann, S., Falk, A., \& Wibral, M. (2012). Promotions and incentives: The case of multistage elimination tournaments. Journal of Labor Economics, 30(1), $149-174$.

[2] Bayer, R. C., \& Renou, L. (2016). Logical omniscience at the laboratory. Journal of Behavioral and Experimental Economics, 64, 41-49.

[3] Brosig-Koch, Jeannette, Timo Heinrich, and Christoph Helbach. "Does truth win when teams reason strategically?." Economics Letters 123.1 (2014): 86-89.

[4] Brosig-Koch, Jeannette, Timo Heinrich, and Christoph Helbach. "Exploring the capability to reason backwards: An experimental study with children, adolescents, and young adults." European Economic Review 74 (2015): 286-302.

[5] Burks, S. V., Carpenter, J. P., Goette, L., \& Rustichini, A. (2009). Cognitive skills affect economic preferences, strategic behavior, and job attachment. Proceedings of the National Academy of Sciences, 106(19), 7745-7750.

[6] Cardella, Eric. "Learning to make better strategic decisions." Journal of Economic Behavior \& Organization 84.1 (2012): 382-392.

[7] Carpenter, Jeffrey, Michael Graham, and Jesse Wolf. "Cognitive ability and strategic sophistication." Games and Economic Behavior 80 (2013): 115-130.

[8] Cueva, C., \& Rustichini, A. (2015). Is financial instability male-driven? Gender and cognitive skills in experimental asset markets. Journal of Economic Behavior \& Organization, 119, 330-344.

[9] Dufwenberg, Martin, Ramya Sundaram, and David J. Butler. "Epiphany in the Game of 21." Journal of Economic Behavior \& Organization 75.2 (2010): 132-143.

[10] Fey, Mark, Richard D. McKelvey, and Thomas R. Palfrey (1996), "An experimental study of constant-sum centipede games," International Journal of Game Theory 25, 269-287.

[11] Flannery, T., \& Roberts, S. (2018). The use of non-monotonic contracts in a single period game: An experimental investigation. Journal of behavioral and experimental economics, 77, 177-185.

[12] Gneezy, Uri, Aldo Rustichini, and Alexander Vostroknutov. "Experience and insight in the race game." K 75.2 (2010): 144-155.

[13] Greiner, B. (2015). Subject pool recruitment procedures: organizing experiments with ORSEE. Journal of the Economic Science Association, 1(1), 114125. 
[14] Hall, Kelly Padden. "A pure test of backward induction." (2009).

[15] Hendriks, A. (2012). SoPHIE-Software platform for human interaction experiments. University of Osnabrueck, working paper.

[16] Hoppe, E. I., \& Schmitz, P. W. (2015). Do sellers offer menus of contracts to separate buyer types? An experimental test of adverse selection theory. Games and Economic Behavior, 89, 17-33.

[17] Johnson, E. J., Camerer, C., Sen, S., \& Rymon, T. (2002). Detecting failures of backward induction: Monitoring information search in sequential bargaining. Journal of Economic Theory, 104(1), 16-47.

[18] McKelvey, R. D., \& Palfrey, T. R. (1992). An experimental study of the centipede game. Econometrica: Journal of the Econometric Society, 803-836.

[19] Neilson, William, Michael Price, and Mikhael Shor. Nudging Backward Induction. No. 2016-31. (2016).

[20] Parsons, Andrew John. "Incentivised Learning In The Game of 21." (2016).

[21] Roomets, Alex. "On limited foresight in games." (2010).

[22] Rosenthal, Robert. "Games of Perfect Information, Predatory Pricing, and the Chain Store Paradox," Journal of Economic Theory, 25, 92-100. (1982) 